\title{
Chromosome positioning is largely unaffected in lymphoblastoid cell lines containing emerin or A-type lamin mutations
}

\author{
K.J. Meaburn*1, N. Levy†, D. Tonioloł and J.M. Bridger"2 \\ "Laboratory of Nuclear and Genomic Health, Cell and Chromosome Biology Group, Division of Biosciences, School of Health Sciences and Social Care, \\ Brunel University, Uxbridge, Middlesex UB8 3PH, U.K., †Inserm U491, Genetique Medicale et Developpement, Faculte de Medecine de la Marseille, \\ 13385 Marseille Cedex 05, France, and ¥Institute of Molecular Genetics-CNR, Via Abbiategrasso 207, Pavia, Italy
}

\begin{abstract}
Gene-poor human chromosomes are reproducibly found at the nuclear periphery in proliferating cells. There are a number of inner nuclear envelope proteins that may have roles in chromosome location and anchorage, e.g. emerin and A-type lamins. In the last decade, a number of diseases associated with tissue degeneration and premature aging have been linked with mutations in lamin A or emerin. These are termed laminopathies, with mutations in emerin causing Emery-Dreifuss muscular dystrophy. Despite highly aberrant nuclear distributions of A-type lamins and emerin in lymphoblastoid cell lines derived from patients with emerin or lamin A mutations, little or no change in chromosome location was detected.
\end{abstract}

\section{Introduction}

Interphase chromosomes are organized into individual territories and are positioned non-randomly within proliferating nuclei according to gene density [1]. Genome organization has been seen to deviate from the norm in cells derived from patients with certain diseases [2].

To date, little is known about which proteins and structures organize chromosome territories into their non-random nuclear positions. However, proteins of the INE [inner NE (nuclear envelope)] and the nuclear lamina have putative roles in targeting and anchoring peripheral chromosomes to the nuclear edge [3], particularly lamin A and emerin, which are complexed [4], with emerin requiring lamin A to become located at the NE [5]. A role in genome organization is supported by the ability of emerin and A-type lamins to bind DNA and chromatin and by cells derived from patient and mouse nucleopathy models having a thin or absent layer of heterochromatin at the NE [2].

Mutations in the genes of emerin and A-type lamins can result in X-EDMD (X-linked Emery-Driefuss muscular dystrophy) and in a set of diseases collectively termed laminopathies respectively. Since A-type lamins and emerin could hypothetically have roles in genome organization, laminopathy and X-EDMD patients could have radically altered chromosome positioning, which could be highly relevant to disease phenotype. To test the role of these NE proteins in genome organization, the positions of selected gene-poor

Key words: A-type lamin, chromosome positioning, genome organization, laminopathy, lymphoblastoid cell, nuclear architecture.

Abbreviations used: A-EDMD, autosomal Emery-Driefuss muscular dystrophy; FISH, fluorescence in situ hybridization; NE, nuclear envelope; INE, inner NE; RT, room temperature; X-EDMD, X-linked EDMD.

1'Present address: Cell Biology of Gene Expression, National Cancer Institute, National Institutes of Health, Bethesda, MD 20892, U.S.A.

${ }^{2}$ To whom correspondence should be addressed (email Joanna.Bridger@brunel.ac.uk). chromosomes normally located at the NE were analysed in lymphoblastoid cells derived from X-EDMD and laminopathy patients and in a normal control cell line. Despite a reduced fraction of cells displaying lamin $\mathrm{A} / \mathrm{C}$ and emerin at the NE in the diseased lymphoblastoid cell lines studied, little or no change in chromosome location was detected.

\section{Materials and methods}

\section{Cell culture}

Lymphoblastoid cell lines were maintained in RPMI 1640 supplemented with $15 \%(\mathrm{v} / \mathrm{v})$ foetal bovine serum and $2 \%$ (v/v) penicillin/streptomycin. All cells were cultured at $37^{\circ} \mathrm{C}$ in a humidified atmosphere containing $5 \% \mathrm{CO}_{2}$. Cell lines employed were FATO (control [6]), ED1 (X-EDMD [7]), LB1520 (X-EDMD [7]), LB751 (X-EDMD [7]), LB304/95 (X-EDMD [7]), LB1334/95 \{A-EDMD (autosomal EDMD) $[8,9]\}, \mathrm{LB} 27 / 95$ (A-EDMD [8]) and 4710 [CMT2B (Charcot Marie Tooth 2B) [10]].

\section{Two-dimensional FISH (fluorescence} in situ hybridization)

Standard two-dimensional FISH was performed [1]. Total chromosome DNA probes (Obiogene/MP Biomedicals, London, U.K.) were employed as per the manufacturer's instructions. Slides were examined using a $\times 100$ oil immersion objective on a Leica fluorescence microscope and images of nuclei were collected with a CCD (charge-coupled-device) camera (Photometrics Sensys, Cambridge, U.K.) using Quips PathVysion, SmartCapture VP V1.4 (Digital Scientific, Cambridge, U.K.; Vysis, Abbott Laboratories, Downers Grove, IL, U.S.A.). Simple erosion analysis was then performed on the captured images as described in $[1,6]$. This 
Figure 1 Indirect immunofluorescence of lamin A/C and emerin in the disease and control cell lines

(A-N) Cultured lymphoblastoid cells stained for the presence of lamin A/C or emerin (green) and counterstained with DAPI (4,6-diamidino-2-phenylindole) (blue). NE rim, NE rim only staining; NE rim + IF, NE rim and internal foci. No homogenous or honeycomb staining was observed in lymphoblastoid cells but was present in primary laminopathy fibroblasts [12]. Scale bar, $10 \mu \mathrm{m}$. $(\mathbf{0}, \mathbf{P})$ Graphical representation of the fraction of lymphoblastoid cells displaying each distribution pattern of lamin $\mathrm{A} / \mathrm{C}(\mathbf{0})$ and emerin $(\mathbf{P})$.
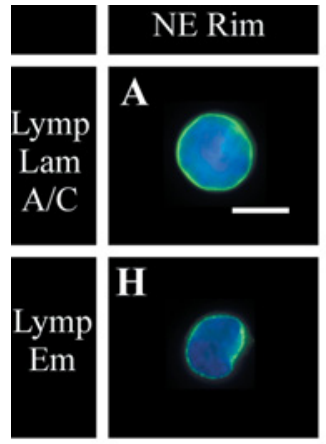

o

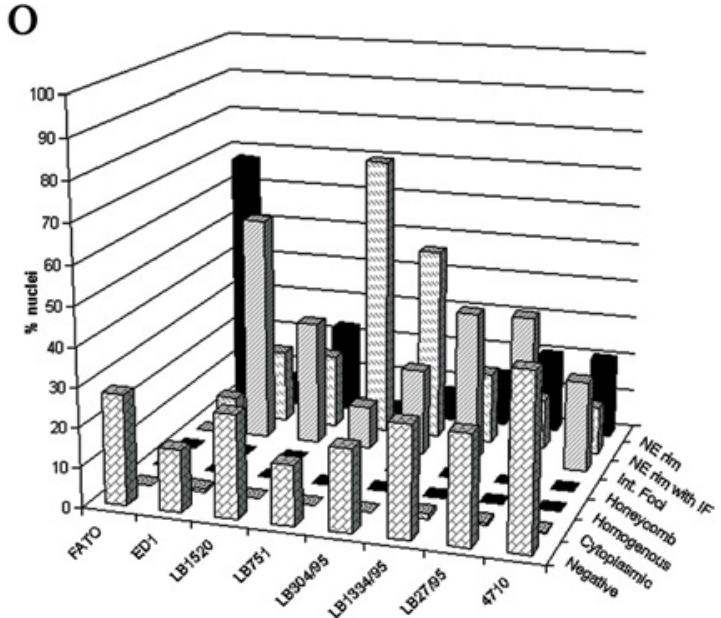

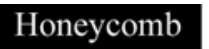
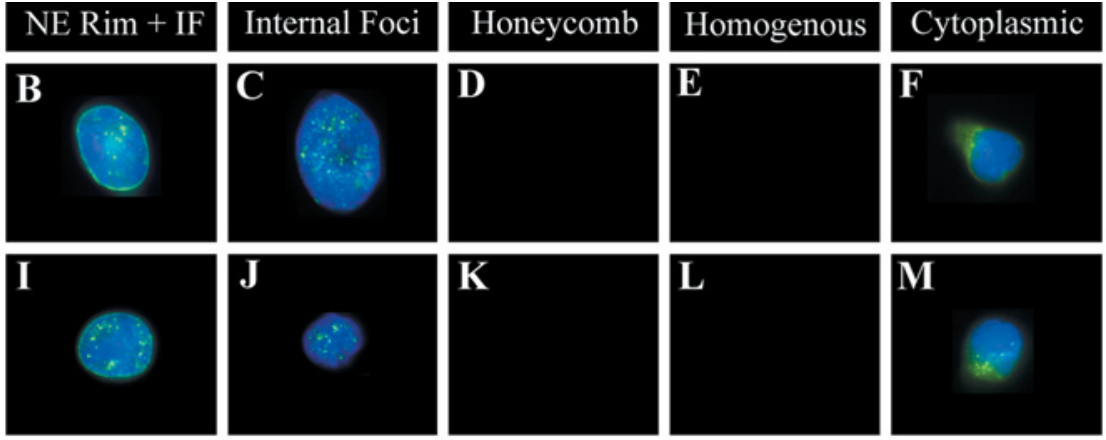

\section{Negative}

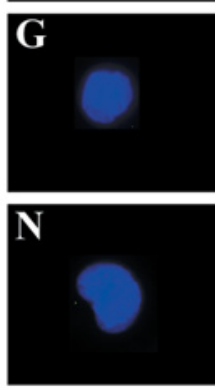

$\mathbf{P}$

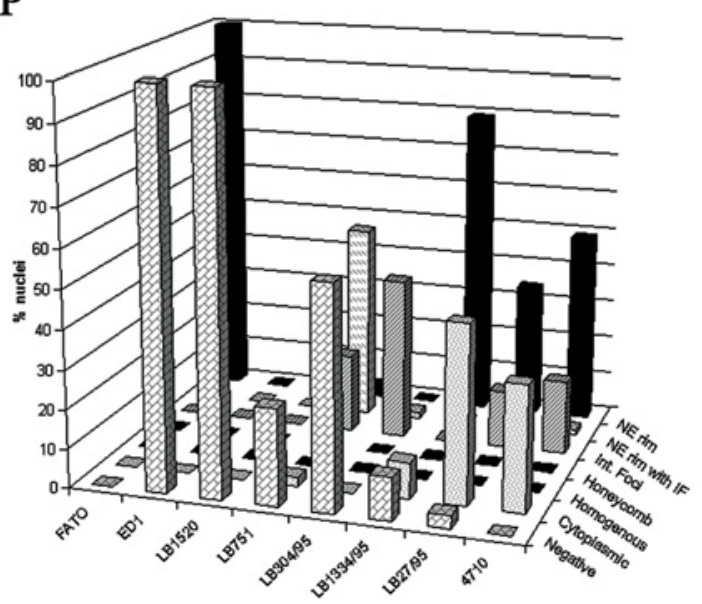

allowed us to determine where in the nuclei the chromosomes were positioned. Statistical analysis was performed with the unpaired, unequal variances, two-tailed Students $t$ test.

\section{Indirect immunofluorescence}

Lymphoblastoid cell suspensions were incubated on poly-Llysine-coated slides (Sigma) for $1 \mathrm{~h}$. The slides were fixed for $10 \mathrm{~min}$ at RT (room temperature) in $4 \%(\mathrm{w} / \mathrm{v})$ paraformaldehyde/PBS. Cells were then incubated in $0.5 \%$ (v/v) Triton X$100 / 0.5 \%$ (w/v) saponin/PBS, for $20 \mathrm{~min}$ at RT, followed by washes in PBS. Primary antibodies used were mouse anti-lamin A/C (Novocastra, Newcastle upon Tyne, U.K.) diluted to 1:10 and mouse anti-emerin (Novocastra) diluted to $1: 20$, followed by secondary antibodies donkey antimouse conjugated to FITC or Cy3 (Jackson Immunoresearch Laboratories, West Grove, PA, U.S.A.; diluted to 1:60).

\section{Results}

We have a library of EBV (Epstein-Barr virus) immortalized lymphoblastoid cells with a range of mutations in the nuclear proteins lamin $\mathrm{A} / \mathrm{C}$ and emerin. To assess whether mutations in lamin $\mathrm{A} / \mathrm{C}$ or emerin have affected lamin $\mathrm{A}$, emerin and chromosome distribution, we performed immunohistochemistry and chromosome positioning analyses.

\section{Laminopathy and X-EDMD lymphoblastoid cells have a highly altered nuclear distribution of A-type lamins and emerin}

The nuclear presence and distribution of A-type lamins and emerin in the lymphoblastoid cell lines was analysed by indirect immunofluorescence. The cells were classified by their staining patterns (Figures $1 \mathrm{~A}-1 \mathrm{~N}$ ). The control cells were $100 \%$ positive for emerin antibody staining and $72 \%$ positive for lamin A/C antibody staining at the NE. However, in the diseased cell lines, lamin A/C was still present at the NE but in a much lower fraction of cells (Figure 1O). The emerin distribution was much more variable with ED1 and LB1520 cells containing no emerin at all, and $1.5-80 \%$ of the other cells displaying emerin at the nuclear periphery, in internal foci and the cytoplasm (Figure 1P).

Interestingly, A-type lamins in laminopathy and $\mathrm{X}$ EDMD lymphoblastoid cell lines were generally localized in 
Table 1 | Positioning of chromosomes 4, 13, 18 and $\mathrm{X}$ in normal and mutant cell lines

$\mathrm{P}$, peripheral position of the chromosome within nuclei; IM, intermediate nuclear position.

\begin{tabular}{|c|c|c|c|c|}
\hline \multirow[b]{2}{*}{ Cell line } & \multicolumn{4}{|c|}{ Chromosome position in each cell line $(n=50-60)$} \\
\hline & Chromosome 4 & Chromosome 13 & Chromosome 18 & Chromosome $\mathrm{X}$ \\
\hline FATO & $P$ & $P$ & $\mathrm{P}$ & $P$ \\
\hline ED1 & $P$ & $P$ & $P$ & $P$ \\
\hline LB1520 & $P$ & $\mathrm{P}$ & $P$ & $P$ \\
\hline LB751 & $P$ & $P$ & $P$ & $P$ \\
\hline LB304/95 & $P$ & $\mathrm{P}$ & $P$ & $P$ \\
\hline LB1334/95 & $P$ & IM & $P$ & $P$ \\
\hline LB27/95 & $P$ & IM & $\mathrm{P}$ & $P$ \\
\hline 4710 & $P$ & $P$ & $P$ & $P$ \\
\hline
\end{tabular}

internal nuclear foci, with a lower fraction of cells displaying A-type lamin only at the nuclear periphery (Figure 1O). These data imply that mutations in either lamin $\mathrm{A} / \mathrm{C}$ or emerin genes have led to a reduced ability of either the A-type lamins or emerin to locate to the $\mathrm{NE}$, leading to an increase in lamin $\mathrm{A} / \mathrm{C}$ and emerin aggregates within nuclei and of emerin in the cytoplasm, and also in emerinnegative cells. Cell lines ED1 and LB1520 contain cells that are negative for both A-type lamins and emerin, suggesting that neither of these proteins is essential for proliferation in these lymphoblastoid cells. Thus all the disease cell lines studied had an altered distribution or presence of these two proteins.

\section{Genome organization is unaffected by A-type lamin and emerin mutations in lymphoblastoid cells}

We are testing the hypothesis that A-type lamins and/or emerin are involved in translocating and anchoring chromosomes to the NE. To do this, the nuclear localization of the gene-poor human chromosomes 4, 13, 18 and $\mathrm{X}$ was analysed. The results are summarized in Table 1 . Human chromosomes 4, 13, 18 and $\mathrm{X}$ were found to be peripheral in all lymphoblastoid cell lines studied, with the exception of chromosome 13 in two A-EDMD cell lines with the same mutation (LB1334/95 and LB27/95) $(P<0.0005)$. These cell lines do have, however, cells without A-type lamins at the nuclear periphery when emerin is present. The $\mathrm{Arg}^{527}$ mutation in LB1334/95 and LB27/95 is within a DNA- and chromatin-binding domain [11], which maybe responsible for the movement of chromosome 13 away from the nuclear periphery.

\section{Discussion}

The lymphoblastoid cell lines showed very little change in the position of chromosomes despite the hugely altered distribution of A-type lamins and emerin in their nuclei.
The control lymphoblastoid cell line was only $72 \%$ positive for A-type lamins, suggesting that, at least in a subset of cells, A-type lamins are not necessary for chromosome location in these cells. This is even more striking in the 4710 cell line, which has $44 \%$ of cells negative for A-type lamins. Furthermore, there are lymphoblastoid cells that are positive for emerin located at the NE and negative for A-type lamins. This implies that lamin A may not be required for emerin's location at the NE in lymphoblastoid cells.

We thank Paul Perry and Wendy Bickmore (Medical Research Council, HGU, Edinburgh, U.K.) for use of the erosion analysis script and Helen Cox (CCBG, Brunel University, London, U.K.) for technical assistance.

\section{References}

1 Boyle, S., Gilchrist, S., Bridger, J.M., Mahy, N.L., Ellis, J.A. and Bickmore, W.A. (2001) Hum. Mol. Genet. 10, 211-219

2 Foster, H.A. and Bridger, I.M. (2005) Chromosoma, in the press

3 Bridger, J.M. and Bickmore, W.A. (1998) Trends Genet. 14, 403-409

4 Clements, L., Manilal, S., Love, D.R. and Morris, G.E. (2000) Biochem. Biophys. Res. Commun. 267, 709-714

5 Vaughan, O.A., Alvarez-Reyes, M., Bridger, J.M., Broers, J.L.V., Ramaekers, F.C.S., Wehnert, M., Morris, G.M., Whitfield, W.G.F. and Hutchison, C.J. (2001) J. Cell Sci. 114, 2577-2590

6 Croft, J.A., Bridger, J.M., Boyle, S., Perry, P., Teague, P. and Bickmore, W.A. (1999) J. Cell Biol. 145, 1119-1131

7 Bione, S., Maestrini, E., Rivella, S., Mancini, M., Regis, S., Romeo, G. and Toniolo, D. (1994) Nat. Genet. 8, 323-327

8 Bonne, G., Di Barletta, M.R., Varnous, S., Bécane, H.-M., Hammouda, E.-H., Merlini, L., Muntoni, F., Greenberg, C.R., Gary, F., Urtizberea, J.-A. et al. (1999) Nat. Genet. 21, 285-288

9 Raffaele di Barletta, M., Ricci, E., Galluzzi, G., Tonali, P., Mora, M., Morandi, L., Romorini, A., Voit, T., Orstavik, K.H., Merlini, L. et al. (2000) Am. J. Hum. Genet. 66, 1407-1412

10 De Sandre-Giovannoli, A., Chaouch, M., Kozlov, S., Vallat, J.-M., Tazir, M., Kassouri, N., Szepetowski, P., Hammadouche, T., Vandenberghe, A., Stewart, C.L. et al. (2002) Am. J. Genet. 70, 726-736

11 Stierle, V., Couprie, J., Ostlund, C., Krimm, I., Zinn-Justin, S., Hossenlopp, P., Worman, H.J., Courvalin, J.C. and Duband-Goulet, I. (2003) Biochemistry 42, 4819-4828

12 Meaburn, K.M. (2005) Ph.D. thesis, Brunel University

Received 5 July 2005 